\title{
The new trend in the treatment of experimental cryptosporidiosis and the resulting intestinal dysplasia
}

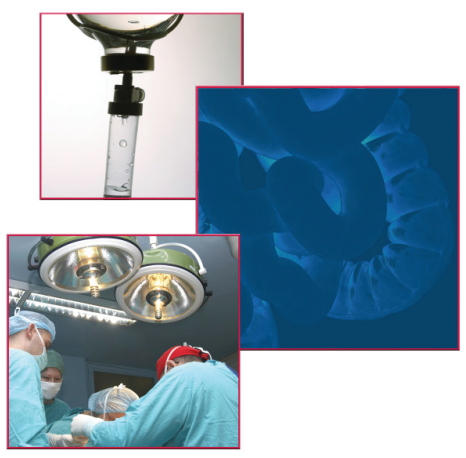

\author{
Nahed E Mostafa ${ }^{1}$, Enas F Abdel Hamed ${ }^{1}$, Eman M Fawzy ${ }^{1}$, Rabab S Zalat ${ }^{2}$, Hayam E \\ Rashed $^{3}$ \& Salem Y Mohamed*,4 \\ ${ }^{1}$ Department of Medical Parasitology, Faculty of Medicine, Zagazig University, Zagazig, Egypt \\ ${ }^{2}$ Department of Medical Parasitology, Theodor Bilharz Research Institute, Cairo, Egypt \\ ${ }^{3}$ Department of Pathology, Faculty of Medicine, Zagazig University, Zagazig, Egypt \\ ${ }^{4}$ Department of Internal Medicine, Gastroenterology \& Hepatology Unit, Zagazig University, Zagazig, Egypt \\ *Author for correspondence: Tel.: +20 114780 5292; Fax: +20 552351 567; salemyousefmohamed@gmail.com
}

\begin{abstract}
Aim: Cryptosporidiosis causes colon dysplasia. This research aimed to evaluate the effect of a novel combination between artesunate and nitazoxanide on intensity of infection and the resulting intestinal dysplasia. Materials \& methods: Subjects were divided into five groups. Artesunate was used alone, then combined with nitazoxanide. Results: The highest efficacy in reducing oocyst shedding obtained from the combined therapy $(68.5,75.9,99 \%)$ after 7,14 and 21 days. The histopathology of infected colonic mucosa showed marked improvement and low-grade of dysplasia in the infected and treated group with the combined therapy. The immunohistochemistry of the same group revealed mild dysplastic changes in colonic epithelium without nuclear expression for cyclin D1. Conclusion: These results give hope for treatment of Cryptosporidium and improving intestinal dysplasia.
\end{abstract}

First draft submitted: 12 May 2018; Accepted for publication: 30 August 2018; Published online: 20 November 2018

Keywords: artesunate $\bullet$ colon dysplasia • Cryptosporidiosis • immunohistochemistry

Cryptosporidiosis is a gastrointestinal infection caused by Cryptosporidium species. It is transmitted by direct fecal contamination or waterborne routes causing diarrhea [1]. In immunocompromised individuals, it is life-threatening as it extends from its habitat; the small intestine and extraintestinal organs such as the biliary tract, lungs and pancreas. Furthermore, there is no fully effective drug treatment [2]. Some studies suggest that infection with Cryptosporidium parvum can lead to digestive carcinogenesis in humans [3,4], stating that C. parvum can be propagated in immunosuppressed mice causing several pathological changes in gastrointestinal epithelial cells, especially in the immunocompromised host. This infection may result in a higher risk of developing colorectal malignancy [5]. Certad et al. [6] confirmed the ability of $C$. parvum isolated from human origin to cause gastrointestinal and biliary adenocarcinomas in an experimental model, which was previously evidenced by Certad et al. [7] who proved the direct role of this parasite in the induction of digestive cancer. The current optimal therapy for cryptosporidiosis is nitazoxanide (NTZ) [8]. NTZ is the most common drug for treating cryptosporidiosis in humans. NTZ, a nitrothiazole benzamide compound, has proved to be effective against a wide variety of protozoa and helminths [9]. Although NTZ has been used for cryptosporidiosis in immunocompetent children and adults, it has limited efficacy in children and has no effect in immunocompromised individuals [10]. Available therapies for cryptosporidiosis are imperfect. Artemisia annua is a plant used in traditional Chinese medicine [11]. It was confirmed that artemisinin exhibits high efficacy against several stages of Plasmodium and other protozoan parasites [12]. Apicomplexan parasites are complex unicellular eukaryotes; they are the causal agents of malaria, toxoplasmosis and cryptosporidiosis [13]. The parasites in this phylum have an infective stage that enters the host via its apical complex. The life cycles of these parasites contain asexual and sexual reproduction. However, all these parasites invade cells and have to adapt to the intracellular environment of their hosts [14]. Artemisinins have broad anticancer effects, they reduce cell proliferation, angiogenesis and trigger apoptosis [15]. The Chinese government's program reported the anticancer properties of artemisinins [16]. Because artemisinin gives a high efficacy against malaria and cancer as well, we pre-

Future Medicine 


\begin{tabular}{|c|c|c|c|c|c|}
\hline Variable & 7 days & 14 days & 21 days & Test & $p$ \\
\hline Control: mean \pm SD & $76,536 \pm 4686$ & $78,913 \pm 1675$ & $81,847 \pm 2215$ & 3.578 & 0.06 (NS) \\
\hline Nanozoxide: mean \pm SD & $67,186 \pm 3706$ & $70,327 \pm 4378$ & $75,167 \pm 2218$ & 6.411 & $0.01(S)$ \\
\hline Artesunate: mean \pm SD & $49,405 \pm 9180$ & $36,277 \pm 5137$ & $32,037 \pm 3948$ & 7.761 & $0.03(S)$ \\
\hline Combined: mean \pm SD range & $24,039 \pm 4153$ & $18,962 \pm 2397$ & $809.9 \pm 311.7$ & 103.6 & $<0.001$ (HS) \\
\hline P\#1: & 0.007 & 0.01 & 0.002 & & \\
\hline P\#2: & $<0.001$ & $<0.001$ & $<0.001$ & & \\
\hline P\#3: & $<0.001$ & $<0.001$ & $<0.001$ & & \\
\hline
\end{tabular}

\begin{tabular}{|c|c|c|c|c|}
\hline \multirow[t]{2}{*}{ Groups } & \multirow[t]{2}{*}{ Treatment } & \multicolumn{3}{|c|}{ Percent efficacy at different days } \\
\hline & & 7 days & 14 days & 21 days \\
\hline 2 & Nanozoxide & 12.2 & 10.8 & 8.2 \\
\hline 3 & Artesunate & 35.4 & 54 & 60.8 \\
\hline 4 & Nanozoxide + artesunate & 68.5 & 75.9 & 99 \\
\hline
\end{tabular}

sumed that it may give an effect against $C$. parvum infection and the resulting intestinal dysplasia. Cell proliferation in certain tissues can be assessed by immunohistochemistry (IHC) measurement of the proliferation-associated antigen expression. Overexpression of D1 has been observed in many tumors. It promotes cell proliferation and differentiation through shortening the G1/S transition. A significant proportion of dysplasia contains molecular abnormalities that may result in cyclin D1 overexpression [17]. Cyclin D1 protein overexpression has been reported in colon carcinoma cell lines, in 30-46\% of primary colorectal cancers [18]. (Bartkova et al.) 1994 Cyclin D1 is a good and useful marker for the detection of intestinal dysplasia [19]. In this research C. parvum obtained from a human was processed for DNA extraction and PCR amplification of C. Parvum then used for infection. The therapeutic benefit of artesunate was evaluated parasitologically, histopathologically and immunohistochemically (cyclin D1).

\section{Materials \& methods \\ The animal}

This study was carried out on laboratory-bred Swiss albino female mice $(n=50)$ weighing about $20 \mathrm{~g}$. Examining their stools for three consecutive days using the formol-ether concentration method [20] and modified ZiehlNeelsen technique [21] to exclude any parasitic infection. The experiment design contains five groups of ten mice each. Animals are immunocompromised: group 1: infected with Cryptosporidium oocysts; group 2: infected then treated with nanozoxide; group 3: infected then treated with artesunate; group 4: infected then treated with an combination of artesunate and nanozoxide; group 5: normal control. The administration doses of the drug started 7 days postinfection (PI) and persisted for 7 days. Fecal samples were collected from each mouse of treated and control groups on 7,14 and 21 days PI and oocysts were counted as described.

Immunosuppression was carried out by giving the mice dexamethasone orally at a dose of $0.25 \mu \mathrm{g} / \mathrm{g} / \mathrm{day}$ for 14 successive days prior to inoculation with Cryptosporidium oocysts and throughout the experiment [22].

Cryptosporidium oocysts were obtained from infected humans in Zagazig University Hospital. Samples were concentrated by modified formalin-ether sedimentation technique [20]. The stool samples were examined by modified Ziehl-Neelsen staining method for confirming the presence of oocysts [21]. Part of the samples was frozen for molecular studies. Other parts of positive samples were purified according to Arrowood and Sterling and preserved in $2.5 \%$ potassium dichromate $\left(\mathrm{K}_{2} \mathrm{Cr}_{2} \mathrm{O}_{7}\right)$ [24]. The number of Cryptosporidium oocysts was determined [25]. 


\section{Molecular identification of C.parvum}

Part of the samples was processed for detection of Cryptosporidium copro-DNA via DNA extraction by isolation of DNA from the fecal sample using (QIAamp DNA Stool Mini Kit QIAGEN, Hilden, Germany). According to the manufacturers' protocol. Amplification of extracted DNA was done using Quantification of Cryptosporidium $18 S$ Ribosomal gene for general laboratory and research use (Figure 1).

\section{The infection}

All mice groups except the control were inoculated intraesophageal with $10^{3}$ Cryptosporidium oocysts [26]. The immunosuppressed groups were inoculated after 15 days of dexamethasone therapy [27]. Tuberculin syringe connected to a polyethylene tube was used.

\section{The drugs}

Nanazoxide Circle 75 Pkwy \#1120, Atlanta, GA 30339, USA (Medizen Pharmaceutical Industries from Utopia Pharmaceuticals) was given orally in a dose of $500 \mathrm{mg} / \mathrm{kg}$ body weight twice daily for five consecutive days [28]. Artesunate (Ipca Laboratories, Kandivli Ind. Estate, Ratlam, India) was given orally in a dose of $200 \mathrm{mg} / \mathrm{kg}$ body weight for five consecutive days. The combination of drugs used was given in a dose of $250 \mathrm{mg} / \mathrm{kg}$ body weight for nanazoxide and $100 \mathrm{mg} / \mathrm{kg}$ body weight for artesunate.

\section{Assessment of the infection \& the drug effect}

\section{Stool examination}

Fecal specimens were collected from mice on days 7, 14 and 21 for monitoring oocyst shedding using modified Ziehl-Neelsen stain [21]. The number of oocysts was calculated according to the following equation (total number of oocysts counted $\times$ dilution factor) / (tested stool volume in $\mathrm{g}$ ). The efficacies of drugs were assessed by comparing the median numbers of oocysts present in treated opposed to infected control mice. Moreover, the reduction percentages in the median number of oocysts in treated opposed to infected control mice were calculated. Efficacy of selected drugs against Cryptosporidium oocysts was calculated as per the formula:

$\%$ Efficacy $=($ Total oocysts before treatment - Total oocysts after treatment $)$. Total oocysts before treatment $\times 100$.

\section{Histopathological examination}

Part of the terminal ileum was removed from each mouse, fixed in 10\% neutral formalin then embedded in paraffin. Sections of $4 \mu \mathrm{m}$ thickness were obtained and stained with hematoxylin and eosin.

\section{Immunohistochemistry (cyclin D1)}

We used the EnVision (GA, USA) method for immunohistochemical staining. Tissue sections $(3-5 \mu \mathrm{m})$ were deparaffinized in xylene and rehydrated in a graded alcohol. Slides were incubated for $10 \mathrm{~min}$ in 3\% hydrogen peroxide to block endogenous peroxidase activity. Dako target retrieval solution ( $\mathrm{pH}$ 6.0) was used for 20 min. Antibody binding was detected by Dako's HRP Envision Kit (Dako Cytomation, Glostrup, Denmark). Tissue samples were incubated with primary antibody (anticyclin D1 antibody [SP4], ab16663, Abcam, Cambridge, UK, diluted in 1/100 PBS) for $1 \mathrm{~h}$. Human colon cancer tissue samples were used as positive control. The areas highly stained were graded based on the staining intensity as follows: grade 0 , the total absence of staining or $<5 \%$ of cells stained; grade 1, mild-to-moderate nuclear staining ( $5-50 \%$ cells stained); grade 2 , strong nuclear staining ( $>50 \%$ cells stained) [29].

\section{Ethical \& regulatory guidelines}

An informed consent was taken from all patients before taking fecal samples. The study was approved by the Research Ethics Committee, Faculty of Medicine, and Zagazig University. The experimental animal studies were conducted according to internationally valid guidelines and maintained in SBSP animal house of TBRI.

\section{Statistical analysis}

Data were analyzed by SPSS software version 19. Test one way ANOVA was used to calculate the significance between all groups but independent $t$-test used between every two groups. The results were considered statistically significant if the p-value was $<0.05$. 


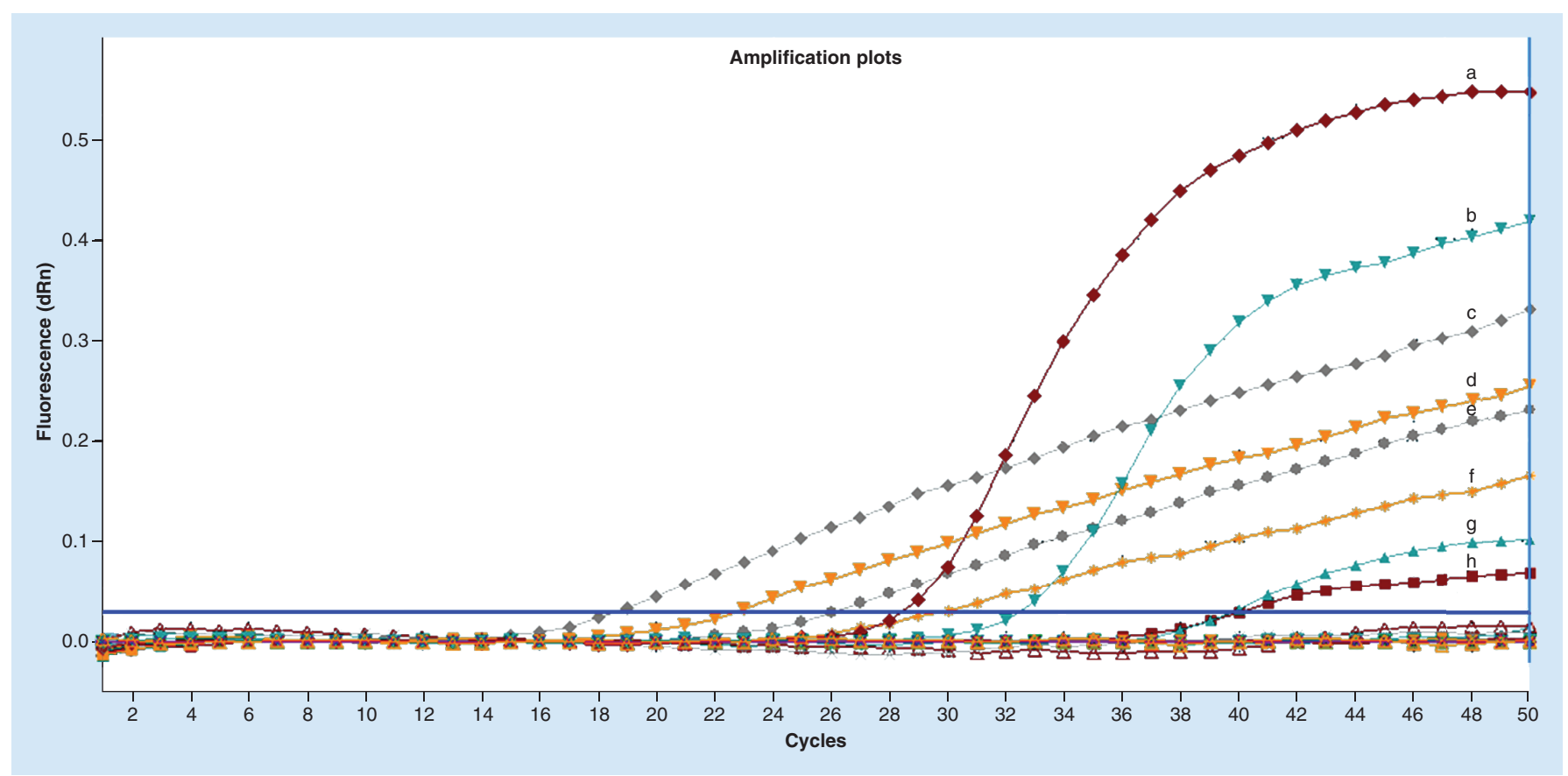

Figure 1. Amplification plots showing results of stool samples by real-time PCR assay for determination of Cryptosporidium parvum species. Plots $(\mathbf{g}, \mathbf{h})$ have fluorescent values above the threshold line 'horizontal blue line' so considered positive samples but with different $\mathrm{CT}$ values. Plots $(\mathbf{a}, \mathbf{b})$ were ACTB controls while the remaining plots were amplified standards. $\mathrm{N}$ were negative samples.

\section{Results}

When comparing oocyst shedding among the immunosuppressed groups on days 7, 14 and 21 to control group, it showed a slight decrease in groups treated with nanozoxide $(67,186 \pm 3706,70,327 \pm 4378,75,167 \pm 2218)$ while groups treated with artesunate showed more decrease $(49,405 \pm 9180 ; 36,277 \pm 5137 ; 32,037 \pm 3948)$. But the combined therapy of artesunate and nanozoxide gave the least mean number of oocysts shedding $(24,039 \pm 4153$; $18,962 \pm 2397 ; 809.9 \pm 311.7)$. There was a significant difference between control and groups treated with at different measurement timings. This difference was highly significant between control and combined treated groups (Table 1). According to percent efficacy of used drugs on days 7, 14 and 21 and, consequently, nanozoxide was at $(12.2,10.8,8.2)$ percent efficacy and artesunate was $(35.4,54,60.8)$ percent efficacy. Finally, the combined therapy of artesunate and nanozoxide gave the highest efficacy in oocyst reduction among the immunosuppressed groups $(68.5,75.9,99 \%$; Table 1).

\section{Discussion}

Cryptosporidium is one of the five leading causes of diarrheal disease in young children worldwide and also causes morbidity in immunocompromised patients [30]. NTZ is partially effective against cryptosporidiosis and the results in AIDS patients remain disappointing [31] in addition to its dose-dependent toxicity [32]. So, a substitute for this drug is urgently needed.

Cryptosporidium belongs to phylum apicomplexan protists, along with Plasmodium and Toxoplasma [33], and artesunate was used for the treatment of malaria by inhibiting an essential parasite calcium adenosine triphosphatase enzyme [34]. Moreover, artesunate possesses selective anticancer properties. It has a cytotoxic effect against a wide range of cancer in vitro and in vivo. Its action is mediated by artemisinin-induced changes in multiple signaling pathways and interfering simultaneously with multiple hallmarks of cancer [35]. From this point of view, we decided to explore the role of artesunate as treatment of cryptosporidiosis and its effect in improving the resulting intestinal dysplasia of immunocompromised mice.

The combined therapy of artesunate and nanozoxide in our research gave the least mean number of oocysts shedding $(24,039 \pm 4153 ; 18,962 \pm 2397 ; 809.9 \pm 311.7)$ on 7,14 and 21 days consequently when compared with the groups treated with single drug either nanozoxide or artesunate (Table 1). Moreover, the combined therapy gave the highest reduction percentages in oocyst shedding among the immunosuppressed groups (68.5, 
75.9, 99\%) when measured on days 7, 14 and 21 consequently (Table 2). It decreases oocyst shedding from 60 to 20 oocysts $/ 100 \mu \mathrm{l}$ feces. Our results are superior to that of Yin et al. and Masood et al. [36,37] who reported an efficacy in oocyst reduction of 43.05, 58.7 and $64.6 \%$ at 13, 20 and 27 days PI after albendazole treatment, 32.8, 53.3 and 56.6\%, respectively, after metronidazole and 55.04, 68.5 and 79.4\% afterparomomycin. Atorvastatin was used alone and combined with NTZ resulting in percentage reduction from (53.7 and 77.5\%) oocytes shed at 21 days PI, respectively, when compared with the infected untreated group [38]. Our better results may be due to the unique combination used.

The histopathological examination of colonic mucosa of its infected immunocompromised group 1 confirms high-grade dysplasia manifested by marked pleomorphism, the absence of mucin, and frequent mitoses (Figure 2A). However, there was a marked improvement in group 4 that was infected, then treated with a combination of artesunate and nanozoxide. The improvement was declared by low-grade dysplasia that manifested by mild pleomorphism, the absence of mucin, and hyperchromatism, in addition to marked mucosal infiltration by inflammatory cells and improvement of the intestinal mucosa (Figure $2 \mathrm{~B} \& \mathrm{C}$ ). There were several studies that

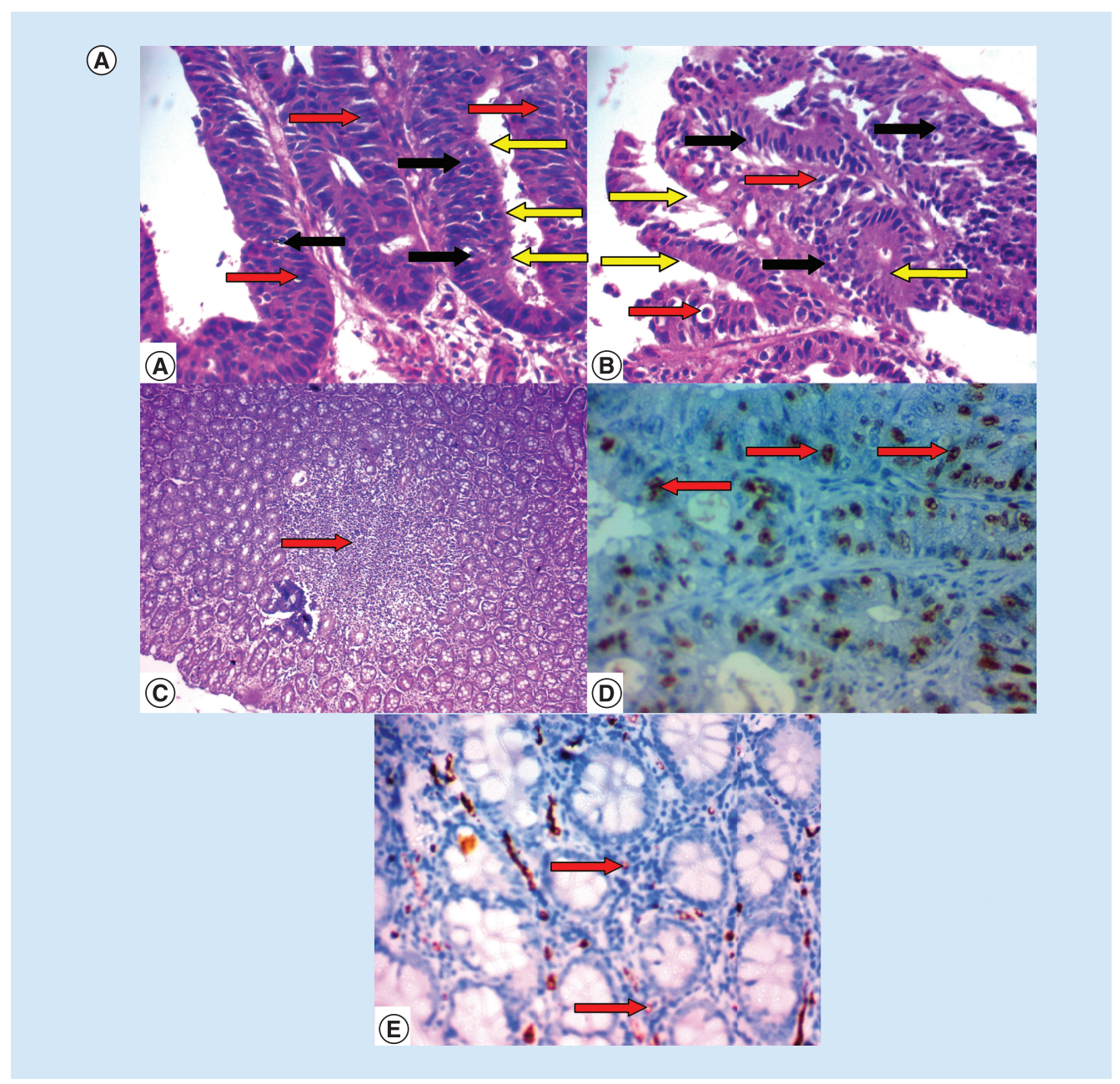

Figure 2. Histopathological and histochemical changes in the studied groups. (A) Infected colonic mucosa of immunocompromised mice (G1) after 21 days postinfection showing high-grade dysplasia in seven out of ten mice and manifested by marked pleomorphism (red arrow), the absence of mucin (yellow arrow) and frequent mitoses (black arrow) (H\&E $\times$ 400); (B) (H\&E $\times$ 400). Infected colonic mucosa in (G4) 21 days postinfection showing low-grade dysplasia manifested by mild pleomorphism (black arrow), the absence of mucin (yellow) and hyperchromatism (red arrows) in addition to marked mucosal infiltration by inflammatory cells (red arrow) (C ) (H\&E x 100). (D) Over nuclear expression of cyclin D1 in the dysplastic colonic epithelium infected by Cryptosporidium parvum (red arrow; immunohistochemical staining $\times 400$ ). (E) Mild dysplastic colonic epithelium infected with $C$. parvum (immunohistochemical staining $\times 400$; red arrow) showed no nuclear expression for cyclin D1. 
(B)
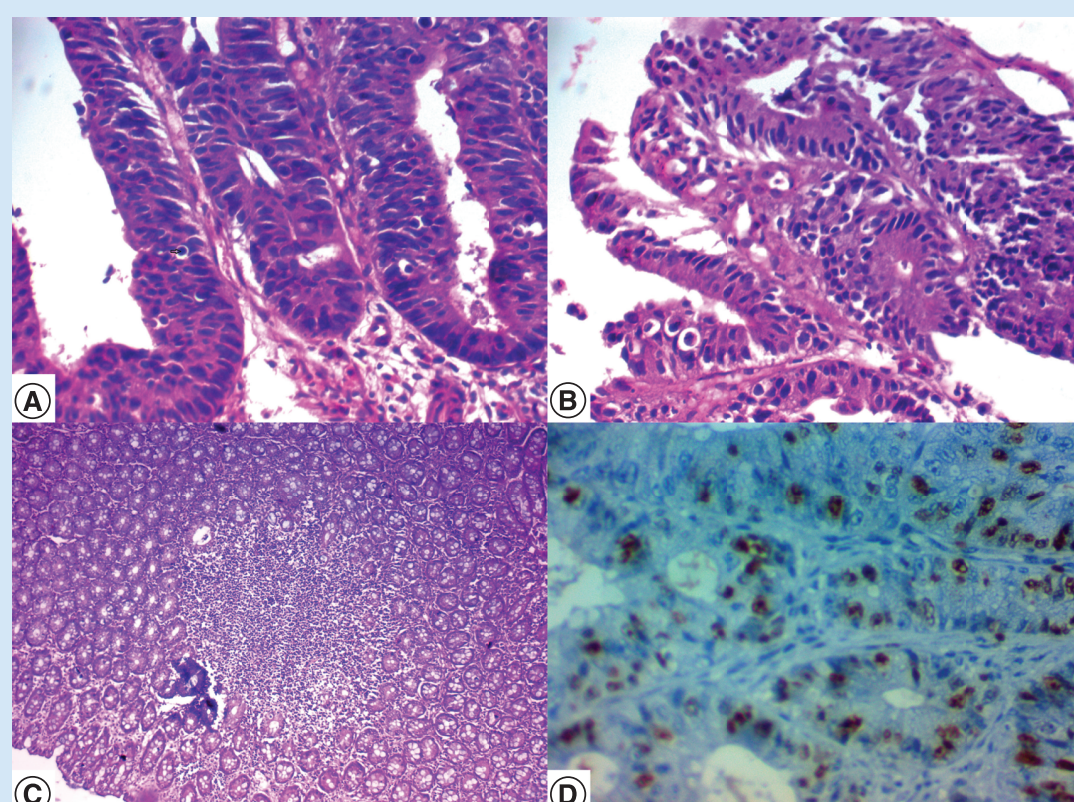

(3)

(C)

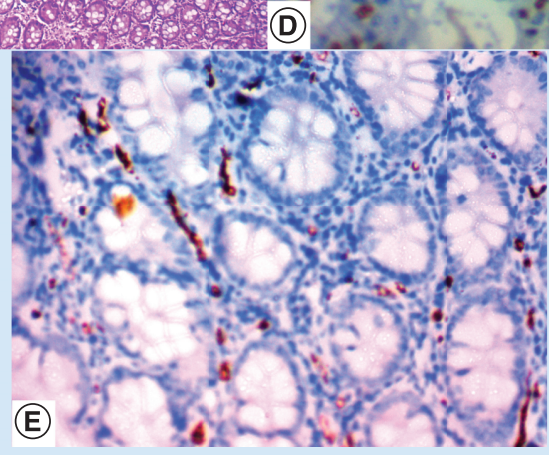

Figure 2. Histopathological and histochemical changes in the studied groups (cont.). (A) Infected colonic mucosa of immunocompromised mice (G1) after 21 days postinfection showing high-grade dysplasia in seven out of ten mice and manifested by marked pleomorphism (red arrow), the absence of mucin (yellow arrow) and frequent mitoses (black arrow) (H\&E $\times 400)$; (B) (H\&E $\times 400)$. Infected colonic mucosa in (G4) 21 days postinfection showing low-grade dysplasia manifested by mild pleomorphism (black arrow), the absence of mucin (yellow) and hyperchromatism (red arrows) in addition to marked mucosal infiltration by inflammatory cells (red arrow) (C) (H\&E x 100). (D) Over nuclear expression of cyclin D1 in the dysplastic colonic epithelium infected by Cryptosporidium parvum (red arrow; immunohistochemical staining $\times 400$ ). (E) Mild dysplastic colonic epithelium infected with $C$. parvum (immunohistochemical staining $\times 400$; red arrow) showed no nuclear expression for cyclin D1.

proved the association between Cryptosporidium infection and induction of digestive neoplasia in mice even in a very low (1-10 oocysts) infection dose [6]. There was a close relationship between cryptosporidiosis and many cases with human colorectal cancer [39]. In addition, Shebl et al. [4] reported that cryptosporidiosis was associated with a rare squamous cell carcinoma of the colon in AIDS cases. This can be explained by Lantier et al. [40] stating that immune cell recruitment and retention in the infected intestine are crucial in the mechanism of protection against C. parvum. Abdou et al. [20] declared that Cryptosporidium may trigger cellular dysplasia in an animal model. The immunohistochemical staining in our research for infected immunocompromised mice group 1 showed high grade dysplastic colonic epithelium with over nuclear expression of cyclin D1 (Figure 2D) when compared with mild dysplastic colonic epithelium in group 4 that infected then treated with combination of artesunate and nanozoxide viewing no nuclear expression for cyclin D1 (Figure 2E). Less severe neoplastic lesions were detected in seven out of ten mice.

This is in accordance with Tan et al. [41] who reported that artemisinin and its semisynthetic have exhibited potential anticancer functions. It demonstrates cytotoxic effects against a wide range of cancer types in vitro and in 
vivo mediated by artemisinin-induced changes in multiple signaling pathways that interfere simultaneously with multiple cancer hallmarks [35]. Finally, the combination of artesunate and nanazoxide showed a synergistic effect by reducing the number of oocysts shed and improving dysplastic changes induced by Cryptosporidium infection in the colon of immunosuppressed mice when compared with that induced by either artesunate or nanazoxide alone.

\section{Conclusion}

The combination of artesunate and nanazoxide showed a synergistic effect by reducing the number of $C$. parvum oocysts shed and improving dysplastic changes induced by Cryptosporidium infection in the colon of immunosuppressed mice when compared with that induced by either artesunate or nanazoxide alone. Our results open a new prospective hope for treatment of Cryptosporidium in immunocompromised individuals in addition to its notable effect in improving intestinal dysplasia caused by this infection.

\section{Summary points}

- Cryptosporidium parvum is a gastrointestinal infection having a devastating effect in the immunocompromised host in addition to the high risk of developing a colorectal malignancy.

- Artesunate gives a high efficacy against protozoan parasites and selective anticancer properties as well.

- Stool examination by (modified Ziehl-Neelsen stain), histopathology, immunohistochemistry with an expression of cyclin D1 was done to evaluate the therapeutic effect of a novel combination between artesunate and nitazoxanide on the intensity of infection and the resulting intestinal dysplasia.

- Combination of artesunate and nanozoxide gave the best therapeutic effect represented by the least mean number of oocysts shedding.

- This combination leads to improvement in histopathological examination of colonic mucosa in immunocompromised mice.

- Immunohistochemical staining of colonic mucosa in target group reveals mild dysplastic changes with no nuclear expression for cyclin D1 when compared with the high-grade dysplasia and over nuclear expression of cyclin D1 in control infected group.

- Combination of artesunate and nanozoxide showed synergistic effects.

Financial \& competing interests disclosure

The authors have no relevant affiliations or financial involvement with any organization or entity with a financial interest in or financial conflict with the subject matter or materials discussed in the manuscript. This includes employment, consultancies, honoraria, stock ownership or options, expert testimony, grants or patents received or pending, or royalties.

No writing assistance was utilized in the production of this manuscript.

\section{Open access}

This work is licensed under the Attribution-NonCommercial-NoDerivatives 4.0 Unported License. To view a copy of this license, visit http://creativecommons.org/licenses/by-nc-nd/4.0/

\section{Ethical conduct of research}

The authors state that they have obtained appropriate institutional review board approval or have followed the principles outlined in the Declaration of Helsinki for all human or animal experimental investigations. In addition, for investigations involving human subjects, informed consent has been obtained from the participants involved.

\section{References}

Papers of special note have been highlighted as: $\bullet$ of interest; $\bullet \bullet$ of considerable interest

1. Desai NT, Sarkar R, Kang G. Cryptosporidiosis: an under-recognized public health problem. Trop. Parasitol. 2, 91-98 (2012).

2. Fayer R. Cryptosporidium: a water-borne zoonotic parasite. Vet. Parasitol. 126, 37-56 (2004).

3. Benamrouz S, Conseil V, Chabé M, Praet M, Audebert C, Blervaque R et al. Cryptosporidium parvum-induced ileocaecal adenocarcinoma and Wnt signaling in a mouse model. Dis. Model Mech. 7, 693-700 (2014).

-• Cryptosporidium is a very dangerous pathogen causing intestinal dysplasia.

4. Shebl FM, Engels EA, Goedert JJ. Opportunistic intestinal infections and risk of colorectal cancer among people with AIDS. AIDS Res. Hum. Retroviruses 28(9), 994-999 (2012). 
-• Cryptosporidium parvum is an opportunistic infection having a risk of colorectal cancer among people with AIDS.

5. Toketo MM. Mouse models of gastrointestinal tumors. Cancer Sci. 97, 355-361 (2006).

6. Certad G, Ngouanesavanh T, Guyot K et al. Cryptosporidium parvum, a potential cause of colic adenocarcinoma. Infect. Agent Cancer 2, 22 (2007).

7. Certad G, Benamrouz S, Guyot K et al. Fulminant cryptosporidiosis after near-drowning: a human Cryptosporidium parvum strain implicated in invasive gastrointestinal adenocarcinoma and cholangiocarcinoma in an experimental model. Appl. Environ. Microbiol. 78, 1746-1751 (2012).

8. Certad G, Creusyc C, Guyot K et al. Fulminant cryptosporidiosis associated with digestive adenocarcinoma in SCID mice infected with C. parvum TUM1 strain. Int. J. Parasitol. 40, 1469-1475 (2010).

9. Hussien SM, Abdella OH, Abu-Hashim AH et al. Comparative study between the effect of nitazoxanide and paromomycin in the treatment of cryptosporidiosis in hospitalized children. J. Egypt Soc. Parasitol. 43, 463-470 (2013).

10. Fox LM, Saravolatz LD. Nitazoxanide: a new thiazolide antiparasitic agent. Clin. Infect. Dis. 40(8), 1173-1180 (2005).

11. Amadi B, Mwiya M, Musuku J et al. Effect of nitazoxanide on morbidity and mortality in Zambian children with cryptosporidiosis: a randomized controlled trial. Lancet 360(9343), 1375-1380 (2002).

12. Klayman DL. Qinghaosu (artemisinin): an antimalarial drug from China. Science 228, 1049-1055 (1985).

-• Malaria and Cryptosporidium are Apicomplexa. As artemisinin had a magic effect on malaria, we presumed that it has an effect on cryptosporidosis.

13. Golenser J, Waknine JH, Krugliak M, Hunt NH, Grau GE. Current perspectives on the mechanism of action of artemisinins. Int. J. Parasitol. 36, 1427-1441 (2006).

14. Mehlhorn H. Encyclopedia of Parasitology (3rd Ed.), Springer (2008). ISBN-978-3-540-48996-2

15. Andreyev AY, Kushnareva YE, Starkov AA. Mitochondrial metabolism of reactive oxygen species. Biochemistry 70(2), $200-214$ (2005).

16. Efferth T, Giaisi M, Merling A, Krammer PH, Weber M. Artesunate induces ROS-mediated apoptosis in doxorubicin-resistant T leukemia cells. PLoS ONE 2(8), e693 (2007).

17. Krishna S, Bustamante L, Haynes RK, Staines HM. Artemisinins: their growing importance in medicine. Trends Pharmacol. Sci. 29(10), 520-527 doi: 10.1016/j.tips (2008).

18. Huang S, Chen CS, Ingber DE. Control of Cyclin D1, p27Kip1, and cell cycle progression in human capillary endothelial cells by cell shape and cytoskeletal tension. Mol. Biol. Cell 9, 3179-3193 (1998).

19. Bartkova J, Lukas J, Strauss M, Bartek J. Cyclin D1 oncoprotein aberrantly accumulates in malignancies of diverse histogenesis. Oncogene 10, 775-778 (1995).

20. Abdou AG, Harba NM, Afifi AF, Elnaidany NF. Assessment of Cryptosporidium parvo infection in immunocompetent and immunocompromised mice and its role in triggering intestinal dysplasia. Int. J. Infect. Dis. 17(8), 593-600 (2013).

21. Waldman E, Tzipori S, Forsyth JRL. Separation of Cryptosporidium species oocysts from feces by using a percoll discontinuous density gradient. J. Clin. Microbiol. 23, 199-200 (1986).

22. Henriksen SA, Pohlenz JF. Staining of cryptosporidia by a modified Ziehl-Neelsen technique. Acta Vet. Scand. 22, $594-596$ (1981).

23. Rehg JE, Hancock ML, Woodmansee DB. Characterization of a dexamethasone-treated rat model of cryptosporidial infection. J. Infect. Dis. 1586, 1406-1407 (1988).

24. Arrowood MJ, Sterling CR. Isolation of Cryptosporidium oocysts and sporozoites using discontinuous sucrose and isopycnicpercoll gradient. J. Parasitol. 73(2), 314-319 (1987).

25. Campbell PN, Current WL. Demonstration of serum antibodies to Cryptosporidium sp. in normal and immunodeficient humans with confirmed infections. J. Clin. Microbiol. 18, 165-169 (1983).

26. Zierdt WS. Concentration and identification of Cryptosporidium sp. by use of a parasite concentrator. J. Clin. Microbiol. 20, 860-861 (1984).

27. Warren CA, Guerrant RL. Clinical disease and pathology. In: Cryptosporidium and Cryptosporidiosis (2nd Ed.). Fayer R, Xiao L (Eds). Taylors \& Francis Group, USA, 235-253 (2008). ISBN 9781420052275

28. Moon HW, Schwartz A, Welch MJ, McCann PP, Runnels PL. Experimental fecal transmission of human cryptosporidia to pigs, and attempted treatment with an ornithine decarboxylase inhibitor. Vet. Pathol. 19, 700-707 (1982).

29. Paget GE, Barnes JM. Evaluation of drug activities. In: Pharmacometrics. Laurence DR, Backarach AL (Eds). Academic Press, London, UK and NY, USA (1964).

30. Ramasubramanian A, Ramani P, Sherlin HJ, Premkumar P, Natesan A, Thiruvengadam C. Immunohistochemical evaluation of oral epithelial dysplasia using cyclin-D1, p27 and p63 expression as predictors of malignant transformation. J. Nat. Sci. 4, 349-358 (2013).

31. Checkley W, White AC, Jaganath D et al. A review of the global burden, novel diagnostics, therapeutics and vaccine targets for Cryptosporidium. Lancet Infect. Dis. 15, 85-94 (2015). 
32. Agnamey P, Djeddi D, Diallo A et al. Childhood cryptosporidiosis: a case report. J. Parasitol. Res. pii: 935625. doi: 10.1155/2010/ (2010) (Epub ahead of print).

33. Gargala G, Le Goff L, Ballet JJ, Favennec L, Stachulski AV, Rossignol JF. Evaluation of new thiazolide/thiadiazole derivatives reveals nitro group-independent efficacy against in vitro development of Cryptosporidium parvum. Antimicrob. Agents Chemother. 54, 1315-1318 (2010).

34. Keithly JS, Langreth SG, Buttle K F, Mannella CA. Electron tomographic and ultrastructural analysis of the Cryptosporidium parvum relict mitochondrion, its associated membranes, and organelles. J. Eukaryot. Microbiol. 52(2), 132-140 (2005).

35. Lisewski AM, Quiros JP, Ng CL et al. Supergenomic network compression and the discovery of EXP1 as a glutathione transferase inhibited by artesunate. Cell 158(4), 916-928 (2014).

36. Yin KW, Xu C, Kalesh KA, He Y et al. Artemisinin as an anticancer drug: recent advances in target profiling and mechanisms of action. Med. Res. Rev. 37(6), 1492-1517 (2017).

-• Gives a recent explanation for the mechanism of action of artemisinin as an anticancer drug.

37. Masood S., Maqbool A., Khan JU, Chaudhry IZ, Anjum AA. Anti-Cryptosporidium activity of albendazole, metronidazole, and paromomycin in experimentally infected cattle. Pakistan J. Zool. 45, 935-940 (2013).

38. Madbouly NT, Hebat SA Yousof, SH El-Sayed, Younis AI, Mohamed SJ. Atorvastatin repurposing for the treatment of cryptosporidiosis in experimentally immunosuppressed mice. Exp. Parasitol. 181, 57-69 (2017).

39. Sulzyc-Bielicka V, Kolodziejczyk L, Jaczewska S, Bielicki D, Kladny J, Safranow K. Prevalence of Cryptosporidium sp. in patients with colorectal cancer. Pol. Przegl. Chir. 84(7), 348-351 (2012).

-. Interesting research concerning the prevalence of Cryptosporidium sp. in patients with colorectal cancer.

40. Lantier L, Lacroix-Lamande S, Potiron L, Metton et al. Intestinal $\mathrm{CD}_{103}{ }^{+}$dendritic cells are key players in the innate immune control of Cryptosporidium parvum infection in neonatal mice. PLoS Pathog. 9, e1003801 (2013).

41. Tan SSL, Ong B, Cheng $\mathrm{C}$ et al. The antimalarial drug artesunate inhibits primary human cultured airway smooth muscle cell proliferation. Am. J. Respir. Cell Mol. Biol. 50(2), 451-458 (2014). 
